\title{
RELAÇÕES ENTRE O DESEMPENHO DOS PROJETOS DE TI E A MATURIDADE EM GESTÃO DE PROJETOS
}

\section{RELATIONSHIP BETWEEN THE IT PROJECTS PERFORMANCE AND PROJECT MANAGEMENT MATURITY}

\author{
Renato de Oliveira Moraes* E-mail: remo@usp.br \\ Fernando José Barbin Laurindo*E-mail: fjblau@usp.br \\ *Universidade de São Paulo (USP), São Paulo, SP
}

Resumo: Este artigo apresenta um estudo sobre as relações entre desempenho de projetos de TI e a maturidade da organização executante em gestão de projetos. A pesquisa foi conduzida por meio de um levantamento com 185 respondentes durante o ano de 2010. O desempenho dos projetos foi avaliado pelas duas primeiras dimensões de Shenhar et al. (2001) - eficiência do projeto e impacto no cliente. A maturidade em gestão de projetos foi avaliada pelo nível de formalização dos processos de gestão de projetos descritos no PMBoK. Os dados foram analisados por meio de procedimentos estatísticos (análise fatorial, alpha de Cronbach, análise de clusters e análise bivariada) e revelaram que (i) que as organizações com maturidade superior têm um desempenho superior em seus projetos e, (ii) diferentes dimensões da maturidade tem impactos distintos no desempenho dos projetos de TI.

Palavras-chave: Desempenho de projetos de TI. Maturidade em gestão de projetos. Gestão de projetos de TI.

\begin{abstract}
This paper presents a study on the relationship between performance of IT projects and the project management maturity of performing organization. The research was conducted through a survey with 185 respondents during the year 2010. The project performance was evaluated through the first two dimensions Shenhar et al (2001) - project efficiency and customer impact. The maturity of project management was assessed by the level of formalization of procedures for project management described in PMBoK. The data were analyzed using statistical procedures (factor analysis, Cronbach's alpha, cluster analysis and bivariate analysis) and revealed that (i) that organizations with higher maturity have a superior performance in their projects, and (ii) different dimensions of maturity has different impacts on the performance of IT projects.
\end{abstract}

Key words: IT projects performance. Pproject management maturity. IT project management.

\section{INTRODUÇÃO}

Nas últimas décadas, a virtualização das atividades e os novos modelos de negócios que ela proporcionou, bem como a coordenação em âmbito mundial de grandes cadeias produtivas caracterizam o que muitos autores passaram a chamar de "Nova Economia" (GEREFFI, 2001).

Ainda segundo Gereffi (2001), há quem denomine esta nova configuração das atividades econômicas de "economia digital", "economia da inovação", "economia 
das redes" ou ainda "economia eletrônica" (e-economy). Para este mesmo autor, a chamada "globalização" é caracterizada pela virtualização e pela coordenação das atividades em âmbito mundial fundamentalmente por meio da utilização de aplicações de Tecnologia da Informação.

A tecnologia da informação (TI) foi. pois, o elemento que viabilizou este novo cenário (HAYES et al., 2008; YASSINE et al., 2004; BENKO; MCFARLAN, 2003; FARREL, 2003; BROWN; GOOLSBEE, 2002; VENKATRAMAN; HENDERSON, 1998), sendo o fator mais visível destas grandes transformações, no qual Castells (1999) usa a expressão "sociedade da informação" para caracterizar os amplos impactos na sociedade. Tapscott (2001) indica como traços distintivos da "nova economia" a perspectiva dos negócios serem baseados em redes e que o conhecimento seria o principal fator de vantagem competitiva.

Portanto, ainda que haja algum tipo de questionamento acerca da extensão dos impactos da tecnologia (PORTER, 2001) fica clara a grande importância que as aplicações de TI (quer sejam sob a forma de produtos ou de serviços) apresentam para as atividades econômicas dos mercados globalizados, em particular para a busca de inovações que permitam as organizações competirem com sucesso (PRAHALAD; KRISHNAN, 2008; BALLOCO et al., 2006).

A ideia de maturidade em gestão de projetos tem despertado grande interesse desde o final da década de 90 (PAULK, 1994). Muito influenciada pelo modelo CMM da Universidade de Carnegie Mellon, os modelos de maturidade em gestão de projetos acenam com a possibilidade de uma consistente melhoria de desempenho dos projetos nas organizações (McGRATH, 1998; GOLDSMITH, 1997; IBBS e KWAK, 1997 e 2000; FINCHER e LEVIN, 1997; REMY,1997; HARTMAN e SKULMOSKI, 1997; KALANTJAKOS, 2001; SCHLICHTER, 2001; MAXIMIANO e RABECHINI, 2002). Ao estudar os modelos de maturidade, a hipótese de que o desempenho dos projetos melhore com o aumento da maturidade da organização em gestão de projetos parece bastante razoável.

Com o objetivo de encontrar alguma evidência empírica desta relação (maturidade $\mathrm{x}$ desempenho de projetos) foi realizada uma pesquisa abordando estas questões em projetos de TI. A coleta de dados aconteceu entre novembro de 2009 e julho de 2010. O método utilizado foi o levantamento com 185 profissionais da área de TI. Este estudo revê o trabalho de Moraes (2004) ampliando a base e o conceito de maturidade usado naquele trabalho em uma base de respondentes maior (este 
assunto também foi discutido em MORAES.; KRUGLIANSKAS, 2010; MORAES; LAURINDO, 2008).

Os dois principais conceitos abordados no estudo foram: (i) maturidade em gestão de projetos e (ii) desempenho de projetos.

A maturidade em gestão de projetos foi abordada de forma bastante simplificada - grau de formalização dos processos de gestão de projetos. Apesar dos modelos de gestão de projetos mais populares (como, por exemplo, OPM3 e PMMM) conceituarem maturidade de forma mais abrangente considerando outros aspectos organizacionais, todos utilizam a formalização dos processos de gestão como parte da maturidade. Os processos de gestão de projetos utilizados na pesquisa são os descritos no PMBoK. Esta escolha deveu-se a grande aceitação deste modelo - PMBoK - no âmbito das empresas brasileiras. A análise dos dados revelou que os elementos da amostra poderiam ser agrupados em três grupos de maturidade (grupo de maturidade inferior, de maturidade média e maturidade superior)

Para medir o desempenho dos projetos, foi empregada uma versão reduzida do modelo multidimensional de Shenhar et all (2001). Foram utilizadas as duas primeiras dimensões do modelo proposto por Shenrar et all (2001), ou seja Eficiência do Projeto e Impacto no Cliente. Assim, o desempenho do projeto foi tratado como um conceito de duas dimensões - Eficiência do Projeto e Impacto no Cliente.

Os elementos da amostra puderam ser agrupados também em três grupos de desempenho (grupo de desempenho inferior, desempenho intermediário e desempenho superior).

O capítulo 2 apresenta a revisão da literatura utilizado no trabalho, o terceiro capítulo descreve a metodologia empregada e os dados colhidos estão no capítulo 4. A análise e discussão dos dados levantados podem ser encontrados no capítulo 5 e as considerações finais, no capítulo 6.

\section{REVISÃO DA LITERATURA}

Neste capítulo são abordados os dois temas principais do artigo: desempenho de projetos e maturidade em gestão de projetos. 


\subsection{Desempenho de projetos}

Baker Muphy e Fisher (1983) afirmam que o sucesso (ou fracasso) do projeto corresponde a percepção que os stakeholders têm dele e que os elementos que afetam a percepção de sucesso não são exatamente os mesmos que afetam a percepção de fracasso. Pinto e Slevin (1986) identificaram duas faces do desempenho do projeto. A face interna, ligada a observância de metas de cisto, prazo e qualidade, está muito mais ligada às ações do gerente e da equipe do projeto. Face externa esta relacionada à figura do usuário através dos indicadores uso e satisfação como produto e resolução do problema que originou o projeto. Esta visão, que sugere duas dimensões do desempenho de projetos, influenciou vários autores (LIM e MOHAMED,1999; COOKE-DAVIES, 2000; BACCARINI,1999; MUNNS, 1997) a trabalharem com abordagens semelhantes: sucesso do projeto e sucesso do produto, sucesso micro e sucesso macro, sucesso do projeto e sucesso da gestão projeto.

Shenhar et al. (2001) propuseram uma visão multidimensional do desempenho dos projetos mais abrangente considerando aspectos de curtíssimo, curto, médio e longo prazo do desempenho. Este modelo possui quatro dimensões conforme mostra a Quadro 1. A importância relativa de cada dimensão na percepção de sucesso varia com o tempo de encerramento do projeto (Figura 1). Enquanto a eficiência tem maior importância na avaliação feita logo após a conclusão do projeto, na avaliação de projetos concluídos a vários anos, sua importância tende a ser muito pequena (MORAES e LAURINDO, 2010).

Quadro 1 - Dimensões do desempenho
\begin{tabular}{|l|l|}
\hline Dimensões do desempenho & Indicadores \\
\hline Eficiência do projeto & Meta de prazo \\
& Meta de orçamento \\
\hline \multirow{5}{*}{ Impacto no consumidor } & $\begin{array}{l}\text { Desempenho funcional } \\
\text { Conformidade às especificações técnicas } \\
\text { Preenchimento das necessidades do cliente } \\
\text { Resolução dos problemas do cliente } \\
\text { Uso do produto pelo cliente } \\
\text { Satisfação do cliente }\end{array}$ \\
\hline Sucesso do negócio & $\begin{array}{l}\text { Sucesso comercial } \\
\text { Aumento ou criação de participação de mercado }\end{array}$ \\
\hline \multirow{3}{*}{ Preparação para o futuro } & $\begin{array}{l}\text { Criação de novo mercado } \\
\text { Criação de nova linha de produto } \\
\text { Desenvolvimento de nova tecnologia }\end{array}$ \\
\hline
\end{tabular}

Fonte: Shenhar et al. (2001) 
Uma diferença marcante entre as propostas apresentadas refere-se à discussão em torno da questão da quantidade de conceitos relacionados ao desempenho. Enquanto alguns (LIM e MOHAMED,1999; COOKE-DAVIES, 2000; BACCARINI,1999; MUNNS 1997) referem-se a dois conceitos distintos -sucesso da administração de projeto (foco no processo de desenvolvimento) e sucesso do projeto (foco no produto resultante do projeto) - outros (SHENHAR et al., 2001; BAKER et al. 1983; PINTO e SLEVIN, 1988) entendem que existe um elemento único em discussão que possui características multidimensionais, em que a relevância de cada dimensão varia com o tempo.

Neste trabalho será adotada a segunda linha - um conceito único de desempenho - por entender que ela fornece uma perspectiva temporal em relação ao desempenho de projetos mais interessante. Serão utilizadas, especificamente, as duas primeiras dimensões deste modelo para caracterizar o desempenho dos projetos - eficiência do projeto e impacto no cliente.

Figura 1 - Dimensões do desempenho de projetos

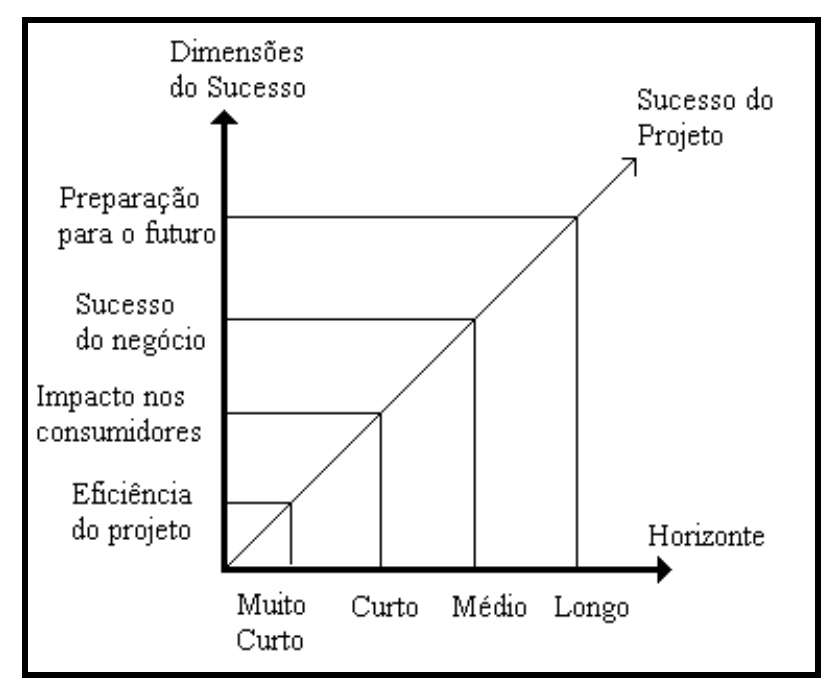

Fonte: Shenhar et al. (2001)

Uma diferença marcante entre as propostas apresentadas refere-se à discussão em torno da questão da quantidade de conceitos relacionados ao desempenho. Enquanto alguns (LIM e MOHAMED,1999; COOKE-DAVIES, 2000; BACCARINI,1999; MUNNS 1997) referem-se a dois conceitos distintos -sucesso da administração de projeto (foco no processo de desenvolvimento) e sucesso do projeto (foco no produto resultante do projeto) - outros (SHENHAR et al., 2001; BAKER et al. 1983; PINTO e SLEVIN, 1988) entendem que existe um elemento 
único em discussão que possui características multidimensionais, em que a relevância de cada dimensão varia com o tempo.

Neste trabalho será adotada a segunda linha - um conceito único de desempenho - por entender que ela fornece uma perspectiva temporal em relação ao desempenho de projetos mais interessante. Serão utilizadas, especificamente, as duas primeiras dimensões deste modelo para caracterizar o desempenho dos projetos - eficiência do projeto e impacto no cliente.

\subsection{Maturidade em gestão de projetos}

A ideia de maturidade em gestão de projetos é fortemente influenciada pelo modelo CMM - Capability Maturity Model da Universidade de Carnegie Mellon (PAULK, 1994) desenvolvido sob o patrocínio do Departamento de Defesa NorteAmericano. Este modelo defini cinco níveis e maturidade em processos de desenvolvimento de software. De maneira geral, os modelos propostos de maturidade em gestão de projetos (GOLDSMIRH, 1997; IBBS e KWAK, 1997 e 2000; REMY, 1997, SCHILICHTER, 2001) usam a estrutura do CMM substituindo os processos de desenvolvimento de software deste modelo pelos processos de gestão de projetos descritos pelo PMBoK.

O conceito de maturidade em gestão de projetos é ligado ao desenvolvimento contínuo de competências especificas em gestão de projetos (KALANTJAKOS, 2001 e SCHLICHTER, 2001), o que sugere a idéia de ser possível estabelecer, de um modo geral, algum tipo de modelo direcionador assim como o PMBoK tem sido.

A ideia de maturidade de processos está associada ao conceito de estabilidade de processos. Processos estáveis são processos livres de variações e que são executadas de forma consistentemente homogênea. A formalização dos processos reflete essa estabilidade como reflete o bordão do modelo ISO 9.000 "faça o que escreve e escreva o que faz" (ANOTINIONI e ROSA, 1995).

Nessa visão, a qualidade de um produto é determinada pela qualidade do processo que o gerou. Assim, a qualidade do processo de desenvolvimento do projeto de software irá condicionar a qualidade do software gerado. Essa é a mesma idéia por trás dos modelos de garantia da qualidade como ISO 9.000-3, CMM e ISO 15.504. Nesses modelos, a qualidade do processo é obtida pela estabilidade dos processos. Dessa forma, quando uma organização inicia sua certificação, os 
auditores procuram verificar se os processos prescritos nesses modelos existem. Além da existência desses processos, são confrontados os seus registros com suas práticas (ANOTINIONI e ROSA, 1995).

Neste trabalho não é utilizado nenhum modelo de maturidade em particular. Esta opção traria o ônus de realizar a avaliação da maturidade dos elementos da amostra e a amostra teria que conter elementos de diferentes níveis de maturidade segundo os critérios do modelo escolhido.

Assim, optou-se por empregar o grau de formalização dos processos de gestão de projetos descritos pelo PMBOK como uma medida da maturidade da organização.

\subsection{Project Management Body of Knowledge - PMBoK}

O PMBoK (Project Management Body of Knowledge, 2004) é o resultado do esforço do PMI (Project Management Institute) em registrar e documentar uma base de conhecimentos para a atividade de Gestão de Projetos. A primeira versão foi publicada em 1984 e revista em 1987 e 1996, as demais edições foram publicadas em 2000, 2004 e 2008. Apesar de esforços similares, como os realizados na Suíça e na Austrália, essa parece ser principal referência em vigor com mais de 450.000 exemplares em circulação. Os vários modelos de maturidade em gestão de projetos utilizam o PMBoK, em maior ou menor grau, como referência conceitual.

O PMBoK descreve um conjunto de processos agrupados em áreas de conhecimento, associados com a Gestão de Projetos (Figura 2). O conhecimento necessário ao bom desempenho de um gerente de projeto, como destaca o PMBoK envolve conhecimentos relacionados a:

Gerência Geral;

Conhecimento e práticas específicas da área em que o projeto está sendo desenvolvido (engenharia civil, computação, farmacologia, etc.); e Gerência de Projetos. 
Figura 2 - Disciplinas gerenciais relacionadas à Gestão de Projetos

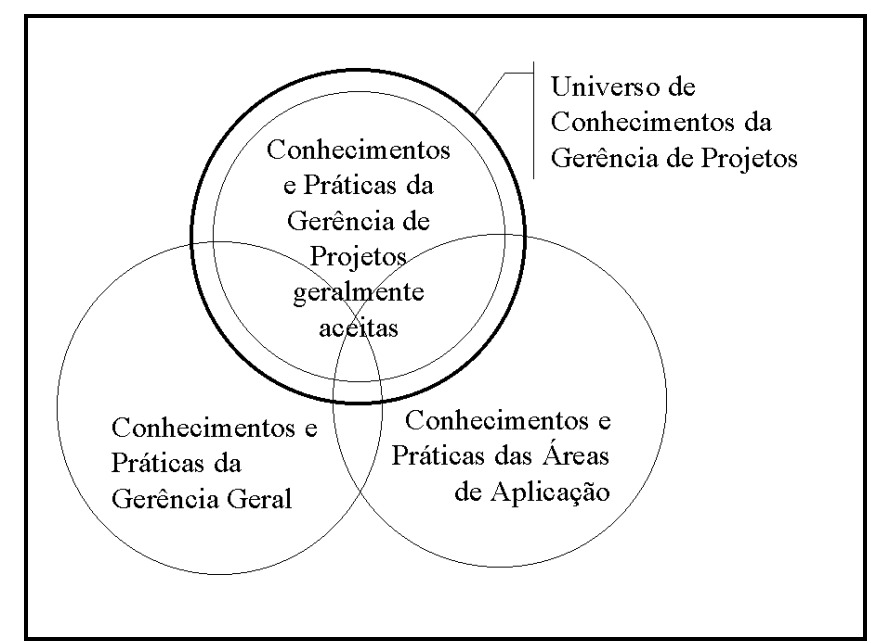

Fonte: PMBoK (2008)

A compilação desses conhecimentos e práticas geralmente aceitas de Gestão de Projetos gerou o PMBoK. Em função de sua proximidade temática, esses conhecimentos foram agrupados em 9 grandes áreas: (i) Gestão da Integração; (ii) Gestão do Escopo; (iii) Gestão do Tempo; (iv) Gestão do Custo; (v) Gestão da Qualidade; (vi) Gestão dos Recursos Humanos; (vii) Gestão das Comunicações;(viii) Gestão dos Riscos; e (ix) Gestão das Aquisições.

Os 44 processos de gestão podem ser classificados, inclusive, em função do ciclo de vida do projeto. Dessa forma, os processos estão divididos em: (i) Processos de iniciação; (ii) Processos de planejamento; (iii) Processos de execução; (iv) Processos de controle; e (v) Processos de encerramento.

A maneira pela qual esses grupos de processos se relacionam está ilustrada na Figura 3. O Quadro 2 contém a descrição de cada um desses grupos.

Figura 3 - Tipos de processos de gestão de projetos

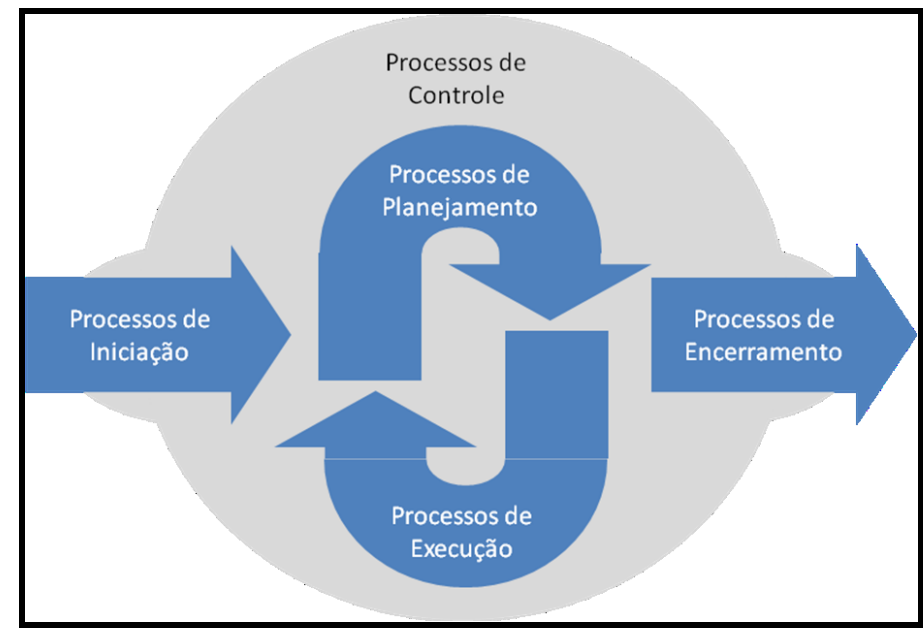

Fonte: $\operatorname{PMBoK}(2008)$ 
Quadro 2 - Áreas de conhecimento em gestão de projetos

\begin{tabular}{|c|c|}
\hline Áreas do Conhecimento & Processos \\
\hline Gestão da Integração & $\begin{array}{l}\text { Termo de abertura do projeto } \\
\text { Declaração preliminar do escopo do projeto } \\
\text { Desenvolver o plano de gerenciamento do projeto } \\
\text { Orientar e gerenciar a execução do projeto } \\
\text { Monitorar e controlar o trabalho do projeto } \\
\text { Controle integrado de mudanças } \\
\text { Encerrar o projeto }\end{array}$ \\
\hline Gestão do Escopo & $\begin{array}{l}\text { Planejamento do escopo } \\
\text { Definição do escopo } \\
\text { Criar EAP } \\
\text { Verificação do escopo } \\
\text { Controle do escopo } \\
\end{array}$ \\
\hline Gestão do Tempo & $\begin{array}{l}\text { Definição da atividade } \\
\text { Seqüenciamento de atividades } \\
\text { Estimativa de recursos da atividade } \\
\text { Estimativa de duração da atividade } \\
\text { Desenvolvimento do cronograma } \\
\text { Controle do cronograma } \\
\end{array}$ \\
\hline Gestão do Custo & $\begin{array}{l}\text { Estimativa de custos } \\
\text { Orçamentação } \\
\text { Controle de custos } \\
\end{array}$ \\
\hline Gestão da Qualidade & $\begin{array}{l}\text { Planejamento da qualidade } \\
\text { Realizar a garantia da qualidade } \\
\text { Realizar o controle da qualidade }\end{array}$ \\
\hline $\begin{array}{l}\text { Gestão dos Recursos } \\
\text { Humanos }\end{array}$ & $\begin{array}{l}\text { Planejamento de recursos humanos } \\
\text { Contratar ou mobilizar a equipe do projeto } \\
\text { Desenvolver a equipe do projeto } \\
\text { Gerenciar a equipe do projeto } \\
\end{array}$ \\
\hline Gestão das Comunicações & $\begin{array}{l}\text { Planejamento das comunicações } \\
\text { Distribuição das informações } \\
\text { Relatório de desempenho } \\
\text { Gerenciar as partes interessadas }\end{array}$ \\
\hline Gestão dos Riscos & $\begin{array}{l}\text { Planejamento do gerenciamento de riscos } \\
\text { Identificação de riscos } \\
\text { Análise qualitativa de riscos } \\
\text { Análise quantitativa de riscos } \\
\text { Planejamento de respostas a riscos } \\
\text { Monitoramento e controle de riscos } \\
\end{array}$ \\
\hline Gestão das Aquisições. & $\begin{array}{l}\text { Planejar compras e aquisições } \\
\text { Planejar contratações } \\
\text { Solicitar respostas de fornecedores } \\
\text { Selecionar fornecedores } \\
\text { Administração de contrato } \\
\text { Encerramento do contrato } \\
\end{array}$ \\
\hline
\end{tabular}

Fonte: PMBoK (2008)

Assim, os processos descritos no PMBoK foram utilizados como as variáveis do levantamento de dados para caracterização do nível de maturidade em gestão de projetos da organização executante, como descrito no item 2.2. 


\section{METODOLOGIA}

Por tentar evidenciar uma relação entre maturidade e desempenho de projetos, esta pesquisa tem caráter explicativo. Porém, possui alguns aspectos descritivos na medida em que os elementos da amostra estudada são agrupados em categorias de maturidade homogênea e dimensões da maturidade em gestão de projetos são identificadas. Foi utilizado o levantamento (survey) como procedimento de coleta de dados. Os dados observados foram analisados através de procedimentos estatísticos uni e multivariados. Para tanto, empregou-se o software SPSS.

A partir da revisão bibliográfica foi elaborado um questionário para coleta de dados com 3 partes:

i. Identificação do entrevistado

ii. Identificação e caracterização da empresa. Nesta parte também é informado o grau de formalização dos processos de gestão de projetos dentro da organização

iii. Caracterização do projeto (desenvolvimento e desempenho). Aqui é feita uma caracterização dos elementos relacionados ao desenvolvimento do projeto e nível do desempenho em termos da eficiência do projeto e do impacto no usuário.

O questionário foi enviado para cerca de 3.500 mil profissionais de $\mathrm{TI}$ que o responderam através de email (com um formulário anexado) e através de um site que hospedou o questionário. Foram obtidos 185 questionários válidos cujo processo de analise incluía:

- Análise fatorial para (a) reduzir os indicadores de desempenho ás duas dimensões de desempenho de projetos do modelo de Shenhar e Dvir (2009) e (b) reduzir o grau de formalização dos processos de gestão projetos a dimensões de maturidade em gestão projetos;

- Análise bivariada entre os fatores gerados na etapa anterior para avaliar a correlação entre maturidade e desempenho;

- Análise de clusters para agrupar os respondentes em função dos diferentes níveis de maturidade em gestão de projetos; e 
- Análise de variância para verificar a existência de diferença (e o padrão desta diferença) de desempenho entre os grupos gerados na etapa anterior.

\section{RESULTADOS}

\subsection{Análise Fatorial do Desempenho}

As oito variáveis relacionadas ao desempenho do projeto foram submetidas à análise fatorial e os resultados foram bastante positivos. A medida de adequação da amostra de Kaiser-Meyer-Olkin (KMO) foi de 0,871. O que, segundo Hair et all (2000), pode ser considerado excelente. Outro sinal positivo foi que nenhuma variável teve comunalidade extraída inferior a 0,5.

Foram extraídos dois fatores que explicam $75,5 \%$ da variação original das variáveis. O segundo fator, cujo eigenvalue (autovalor) é inferior a 1 (mas próximo de 1), foi extraído porque usou-se neste estudo uma definição de desempenho de projetos ter sido adotado o modelo de Shenhar et all (2201), que possui duas dimensões. Nestas condições, os fatores extraídos são consistentes como o modelo de desempenho adotado da literatura.

Tabela 1 - Variação extraída na análise fatorial das variáveis de desempenho

\begin{tabular}{|c|c|c|c|c|c|c|c|}
\hline \multirow[b]{2}{*}{ Component } & \multicolumn{3}{|c|}{ Initial Eigenvalues } & \multicolumn{3}{|c|}{$\begin{array}{c}\text { Extraction Sums of Squared } \\
\text { Loadings }\end{array}$} & \multirow{2}{*}{$\begin{array}{c}\text { Rotation Sums of } \\
\text { Squared Loadings } \\
\text { Total }\end{array}$} \\
\hline & Total & $\begin{array}{c}\% \text { of } \\
\text { Variance }\end{array}$ & $\begin{array}{c}\text { Cumulative } \\
\%\end{array}$ & Total & $\begin{array}{c}\text { \% of } \\
\text { Variance }\end{array}$ & $\begin{array}{l}\text { Cumulati } \\
\text { ve } \%\end{array}$ & \\
\hline 1 & 5,075 & 63,432 & 63,432 & 5,075 & 63,432 & 63,432 & 4,748 \\
\hline 2 & ,962 & 12,021 & 75,453 & ,962 & 12,021 & 75,453 & 3,331 \\
\hline 3 & ,605 & 7,562 & 83,016 & & & & \\
\hline$\vdots$ & $\vdots$ & $\vdots$ & $\vdots$ & & & & \\
\hline 8 & , 145 & 1,812 & 100,000 & & & & \\
\hline
\end{tabular}

Extraction Method: Principal Component Analysis.

Optou-se por uma rotação oblíqua pois a literatura sugere, fortemente, que estas duas dimensões do desempenho de projetos não são independentes. Elas possuem algum grau de correlação. Os resultados estão abaixo. 
O fator 2 corresponde a primeira dimensão do modelo de Shenhar et al (2001) - Eficiência do Projeto, e o fator 1 corresponde a segunda dimensão - Impacto no Usuário.

Tabela 2 - Matriz padrão na análise fatorial das variáveis de desempenho

\begin{tabular}{lcc}
\hline & \multicolumn{2}{c}{ Fator } \\
\hline Preenchimento das necessidades do cliente & 1 & 2 \\
\hline Resolução dos problemas do cliente & 0,934 & $-0,048$ \\
Uso do produto pelo cliente & 0,933 & $-0,077$ \\
Conformidade às especificações técnicas & 0,873 & $-0,082$ \\
Satisfação do cliente & 0,768 & 0,129 \\
Desempenho funcional & 0,690 & 0,235 \\
Meta de orçamento & 0,505 & 0,441 \\
Meta de prazo &,- 0076 & 0,935 \\
\hline
\end{tabular}

A confiabilidade interna, medida usando o Alpha de Crombach, também apresentou excelentes resultados. Ela foi de 0,917 para o fator 1 (Eficiência do Projeto) e de 0,778 para o fator 2 (Impacto no Usuário)

\subsection{Análise Fatorial da Maturidade}

As 44 variáveis relacionadas ao grau de formalização dos processos de gestão de projetos descritos no PMBoK foram submetidas a análise fatorial. $\mathrm{O}$ valor do KMO foi bastante alto $(0,954)$ assim como as comunalidades das variáveis (todas acima de 0,5). 
Tabela 3 - Variação extraída na análise fatorial das variáveis de maturidade em gestão de projeto Total Variance Explained

\begin{tabular}{|c|c|c|c|c|c|c|c|}
\hline \multirow[b]{2}{*}{$\begin{array}{l}\text { Compo } \\
\text { nent }\end{array}$} & \multicolumn{3}{|c|}{ Initial Eigenvalues } & \multicolumn{3}{|c|}{$\begin{array}{c}\text { Extraction Sums of Squared } \\
\text { Loadings }\end{array}$} & \multirow[t]{2}{*}{$\begin{array}{l}\text { Rotation Sums of } \\
\text { Squared Loadings }\end{array}$} \\
\hline & Total & $\begin{array}{c}\% \text { of } \\
\text { Variance }\end{array}$ & $\begin{array}{l}\text { Cumulati } \\
\text { ve } \%\end{array}$ & Total & $\%$ of Variance & $\begin{array}{l}\text { Cumulati } \\
\text { ve } \%\end{array}$ & \\
\hline 1 & 28,428 & 64,609 & 64,609 & 28,428 & 64,609 & 64,609 & 16,178 \\
\hline 2 & 2,659 & 6,043 & 70,652 & 2,659 & 6,043 & 70,652 & 18,352 \\
\hline 3 & 1,685 & 3,829 & 74,481 & 1,685 & 3,829 & 74,481 & 22,349 \\
\hline 4 & 1,177 & 2,675 & 77,156 & 1,177 & 2,675 & 77,156 & 15,093 \\
\hline 5 & 1,036 & 2,355 & 79,511 & 1,036 & 2,355 & 79,511 & 15,581 \\
\hline 6 & 998 & 2,267 & 81,778 & ,998 & 2,267 & 81,778 & 19,069 \\
\hline 7 & 843 & 1,915 & 83,693 & & & & \\
\hline 8 & ,759 & 1,726 & 85,419 & & & & \\
\hline$\vdots$ & $\vdots$ & $\vdots$ & $\vdots$ & & & & \\
\hline 44 &, 016 & ,036 & 100,000 & & & & \\
\hline
\end{tabular}

Extraction Method: Principal Component Analysis

Tabela 4 - Matriz padrão na análise fatorial das variáveis de de maturidade em gestão de projeto

\begin{tabular}{|c|c|c|c|c|c|c|}
\hline & 1 & 2 & 3 & 4 & 5 & 6 \\
\hline Realizar o controle da qualidade &, 594 & & & & & \\
\hline Distribuição das informações &, 555 & & & & & \\
\hline Realizar a garantia da qualidade &, 554 & & & & & \\
\hline Gerenciar as partes interessadas &, 546 & & & & & \\
\hline Planejamento das comunicações &, 534 & & & & & \\
\hline Desenvolver a equipe do projeto &, 510 & & & & & \\
\hline Planejamento da qualidade & ,482 & & & & & \\
\hline Relatório de desempenho & ,454 & & & & & \\
\hline Contratar ou mobilizar a equipe do projeto & ,441 & & & & &,- 404 \\
\hline Selecionar fornecedores & &,- 969 & & & & \\
\hline Solicitar respostas de fornecedores & &,- 907 & & & & \\
\hline Administração de contrato & &,- 895 & & & & \\
\hline Encerramento do contrato & &,- 853 & & & & \\
\hline Planejar contratações & &,- 808 & & & & \\
\hline Planejar compras e aquisições & &,- 804 & & & & \\
\hline Análise qualitativa de riscos & & &,- 961 & & & \\
\hline Planejamento de respostas a riscos & & &,- 923 & & & \\
\hline Identificação de riscos & & &,- 915 & & & \\
\hline Análise quantitativa de riscos & & &,- 892 & & & \\
\hline Planejamento do gerenciamento de riscos & & &,- 890 & & & \\
\hline Monitoramento e controle de riscos & & &,- 870 & & & \\
\hline Estimativa de custos & & & &,- 811 & & \\
\hline Orçamentação & & & &,- 787 & & \\
\hline Controle de custos & & & &,- 725 & & \\
\hline
\end{tabular}


Tabela 4 - Matriz padrão na análise fatorial das variáveis de de maturidade em gestão de projeto (conclusão) 2 3 $4 \quad 5$ 6

Desenvolver a declaração do escopo preliminar do , 750 projeto

Desenvolver o termo de abertura do projeto , 683

Encerrar o projeto , 602

Desenvolver o plano de gerenciamento do projeto , 600

Orientar e gerenciar a execução do projeto , 497

Monitorar e controlar o trabalho do projeto , 484

Definição do escopo

Planejamento do escopo

Verificação do escopo , 437

Controle integrado de mudanças , 414

Criar EAP - estrutura analítica do projeto (WBS)

Controle do escopo

Estimativa de duração da atividade

Seqüenciamento de atividades

Desenvolvimento do cronograma

Definição da atividade

Estimativa de recursos da atividade

Controle do cronograma

Gerenciar a equipe do projeto

Planejamento de recursos humanos

Obs: Foram omitidas as cargas fatoriais inferiores a 0,4

Extraction Method: Principal Component Analysis.

Rotation Method: Oblimin with Kaiser Normalization.

A análise das cargas fatoriais sugere o significado dos fatores extraídos. $O$ fator 1 está relacionado a processos de gestão de diferentes áreas. Como não uma concentração clara de processo de uma área em particular ele será nominado de maturidade geral em gestão de projetos. $\mathrm{O}$ fator 2 está claramente relacionado com os processos de gestão de fornecedores. Portanto, ele será nominado de maturidade em gestão de fornecedores. $\mathrm{O}$ fato das cargas fatoriais serem negativas não é um problema. Nas análises subsequentes será considerado que o valor deste fator deste fator significaria a imaturidade e não a maturidade. $O$ fator 3 refere-se a maturidade em gestão de riscos. O fator 4 envolve processos de gestão da integração e do escopo do projeto e será chamado como tal e o fator 5 refere-se a maturidade em gestão do tempo 
Tabela 5 - Dimensões da maturidade identificadas na análise fatorial

\begin{tabular}{cccc}
\hline Fator & Nome & Sinal & $\begin{array}{c}\text { Alpha de } \\
\text { Cronbach }\end{array}$ \\
\hline 1 & Maturidade da Gestão Geral de Projetos & $(+)$ Maturidade & 0,960 \\
& Maturidade da Gestão de Fornecedores & $(-)$ Imaturidade & 0,970 \\
3 & Maturidade da Gestão de Riscos & $(-)$ Imaturidade & 0,981 \\
4 & Maturidade da Gestão de Custos & $(-)$ Imaturidade & 0,920 \\
5 & Maturidade da Gestão da Integração e do Escopo & $(+)$ Maturidade & 0,952 \\
6 & Maturidade da Gestão do Tempo & $(-)$ Imaturidade & 0,953 \\
\hline
\end{tabular}

\subsection{Análise Bivariada - Correlação entre Desempenho e Maturidade}

A

Tabela mostra a correlação entre as dimensões da maturidade e as dimensões do desempenho através do valor e da significância do coeficiente de correlação de Pearson. A análise dos dados deve considerar que certas dimensões da maturidade foram obtidas através de cargas fatoriais negativas. Assim, a maturidade (o inverso da imaturidade) da gestão de fornecedores está positivamente correlacionada com o desempenho dos projeto. Na verdade, existem evidências de correlação entre todas as dimensões da maturidade em gestão de projetos com as dimensões do desempenho dos projetos. Para verificar o padrão deste relacionamento foram feitas as análises seguintes.

Tabela 6 - Correlação entre as dimensões do desempenho e as dimensões da maturidade

\begin{tabular}{|c|c|c|c|}
\hline \multirow[b]{2}{*}{ Dimensões da Maturidade } & & \multicolumn{2}{|c|}{ Dimensões do Desempenho } \\
\hline & & $\begin{array}{c}\text { Impacto no } \\
\text { Usuário }\end{array}$ & $\begin{array}{c}\text { Eficiência do } \\
\text { Projeto }\end{array}$ \\
\hline (+) Mat Gestão Geral de & Pearson Correlation & ,275 & ,370 \\
\hline Projetos & Sig. (2-tailed) & 000 & ,000 \\
\hline (-) Mat Gestão de & Pearson Correlation &,- 332 &,- 422 \\
\hline Fornecedores & Sig. (2-tailed) & ,000 &, 000 \\
\hline (-) Mat Gestão de Riscos & $\begin{array}{r}\text { Pearson Correlation } \\
\text { Sig. (2-tailed) }\end{array}$ & $\begin{array}{l}-, 343 \\
, 000\end{array}$ & $\begin{array}{l}-, 448 \\
, 000\end{array}$ \\
\hline (-) Mat Gestão de Custos & $\begin{array}{r}\text { Pearson Correlation } \\
\text { Sig. (2-tailed) }\end{array}$ & $\begin{array}{l}-, 336 \\
, 000\end{array}$ & $\begin{array}{l}-, 498 \\
, 000\end{array}$ \\
\hline (+) Mat Gestão da & Pearson Correlation & ,248 & ,403 \\
\hline Integração e do Escopo & Sig. (2-tailed) & 001 & 000 \\
\hline (-) Mat Gestão do Tempo & $\begin{array}{r}\text { Pearson Correlation } \\
\text { Sig. (2-tailed) }\end{array}$ & $\begin{array}{l}-, 198 \\
, 010\end{array}$ & $\begin{array}{l}-, 370 \\
, 000\end{array}$ \\
\hline
\end{tabular}

\subsection{Análise Clusters da Maturidade}

Inicialmente, foi utilizado um método hierárquico de análise de cluster para determinar o número de grupos a serem formados. A análise do roteiro de aglomeração indicou a formação de grupos. A formação dos clusters foi feita através 
do método K-means. O resultado (mostrado na tabela abaixo) é um conjunto de grupos semelhante aos modelos de maturidade em gestão de projetos. São três grupos de maturidade crescente - nos modelos de maturidade são cinco grupos em geral.

Tabela 7 - Resultados da análise de clusters

Centro dos Clusters 23

1 Maturidade Maturidade Maturidade

Fatores empregados na análise de clusters (K-means) Intermediária Inferior Superior

(+) Maturidade da Gestão Geral de Projetos $-0,25138$ $-0,86611$ 0,908532

(-) Maturidade da Gestão de Fornecedores

0,128415

0,912299

$-0,83742$

(-) Maturidade da Gestão de Riscos

0,104822

1,103238

$-0,96844$

(-) Maturidade da Gestão de Custos

0,049962

0,876622

$-0,7403$

(+) Maturidade da Gestão da Integração e do Escopo

0,245226

$-0,96646$

0,55288

(-) Maturidade da Gestão do Tempo

$-0,12398$

1,062802

$-0,73572$

Quantidade de elementos em cada grupo

64

58

73

\subsection{Análise de Variância entre os Clusters da Maturidade}

A comparação da maturidade média entre os 3 grupos através da análise de variância (ANOVA) mostra que o desempenho dos projetos no grupo de maturidade inferior é menor que nos outros grupos, tanto em termos da eficiência como do impacto. Não foi encontrada evidência de diferença do desempenho entre os outros dois grupos (maturidade intermediária e maturidade superior).

Figura 4 - Grupos de maturidade e desempenho de projetos

Revista Produção Online, Florianópolis, SC, v.13, n. 1, p. 61-83, jan./mar. 2013. 
Tabela 8 - ANOVA

\begin{tabular}{clccccc}
\hline & Sum of & Mean & & \\
& & Squares & df & Square & $F$ & Sig. \\
\hline Impacto no & Between Groups & 25,059 & 2 & 12,529 & 15,446 &, 000 \\
Usuário & Within Groups & 136,276 & 168 & 0,811 & & \\
& Total & 161,334 & 170 & & & \\
Eficiência do & Between Groups & 38,776 & 2 & 19,388 & 24,863 &, 000 \\
Projeto & Within Groups & 131,002 & 168 & 0,780 & & \\
& Total & 169,778 & 170 & & & \\
\hline
\end{tabular}

\subsection{Regressão linear entre desempenho e maturidade.}

Com o objetivo de verificar o impacto das dimensões da maturidade sobre o desempenho do projeto. Cabe destacar que, conforme mostra a Tabela, os fatores da maturidade foram gerados através de uma rotação oblíqua (não ortogonal) e que, portanto, existem correlações entre os fatores.

Tabela 9 - Correlações entre as dimensões da maturidade

\begin{tabular}{|c|c|c|c|c|c|c|c|}
\hline & & 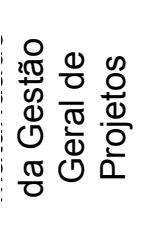 & 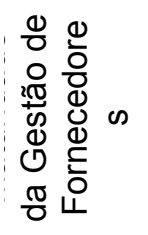 & 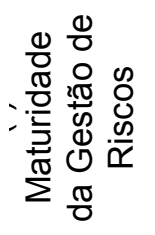 & 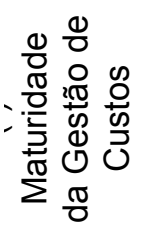 & 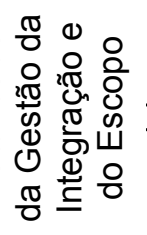 & 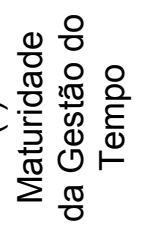 \\
\hline \multirow{3}{*}{$\begin{array}{l}\text { Maturidade da } \\
\text { Gestão Geral de } \\
\text { Projetos }\end{array}$} & $\begin{array}{l}\text { Pearson } \\
\text { Correlation }\end{array}$ & 1 &,- 558 &,- 625 &,- 426 & ,364 &,- 472 \\
\hline & Sig. (2-tailed) & & ,000 & ,000 & ,000 & ,000 & ,000 \\
\hline & $\mathrm{N}$ & 195 & 195 & 195 & 195 & 195 & 195 \\
\hline \multirow{3}{*}{$\begin{array}{l}\text { (-) Maturidade da } \\
\text { Gestão de } \\
\text { Fornecedores }\end{array}$} & $\begin{array}{l}\text { Pearson } \\
\text { Correlation }\end{array}$ &,- 558 & 1 & ,627 & ,557 &,- 425 & ,481 \\
\hline & Sig. (2-tailed) & ,000 & & ,000 & ,000 & ,000 & ,000 \\
\hline & $\mathrm{N}$ & 195 & 195 & 195 & 195 & 195 & 195 \\
\hline \multirow[t]{2}{*}{$\begin{array}{l}\text { (-) Maturidade da } \\
\text { Gestão de Riscos }\end{array}$} & $\begin{array}{l}\text { Pearson } \\
\text { Correlation }\end{array}$ &,- 625 & ,627 & 1 &, 573 &,- 542 & ,648 \\
\hline & $\begin{array}{l}\text { Sig. (2-tailed) } \\
N\end{array}$ & $\begin{array}{r}, 000 \\
195\end{array}$ & $\begin{array}{r}, 000 \\
195\end{array}$ & 195 & $\begin{array}{r}, 000 \\
195\end{array}$ & $\begin{array}{r}, 000 \\
195\end{array}$ & $\begin{array}{r}, 000 \\
195\end{array}$ \\
\hline \multirow[t]{3}{*}{$\begin{array}{l}\text { (-) Maturidade da } \\
\text { Gestão de Custos }\end{array}$} & $\begin{array}{l}\text { Pearson } \\
\text { Correlation }\end{array}$ &,- 426 & ,557 &, 573 & 1 &,- 409 & ,491 \\
\hline & Sig. (2-tailed) & ,000 & ,000 & ,000 & & ,000 & 000 \\
\hline & $\mathrm{N}$ & 195 & 195 & 195 & 195 & 195 & 195 \\
\hline \multirow{3}{*}{$\begin{array}{l}\text { Maturidade da } \\
\text { Gestão da } \\
\text { Integração e do } \\
\text { Escopo }\end{array}$} & $\begin{array}{l}\text { Pearson } \\
\text { Correlation }\end{array}$ & 364, &,- 425 &,- 542 &,- 409 & 1 &,- 609 \\
\hline & Sig. (2-tailed) & ,000 & ,000 & ,000 & ,000 & & ,000 \\
\hline & $\mathrm{N}$ & 195 & 195 & 195 & 195 & 195 & 195 \\
\hline \multirow[t]{3}{*}{$\begin{array}{l}\text { (-) Maturidade da } \\
\text { Gestão do Tempo }\end{array}$} & $\begin{array}{l}\text { Pearson } \\
\text { Correlation }\end{array}$ &,- 472 & ,481 & ,648 &, 491 &,- 609 & - \\
\hline & Sig. (2-tailed) & ,000 & ,000 & ,000 & 000 & ,000 & \\
\hline & $\mathrm{N}$ & 195 & 195 & 195 & 195 & 195 & 195 \\
\hline
\end{tabular}


Inicialmente foi feita uma regressão entre a eficiência do projeto (dimensão do desempenho) com as dimensões d maturidade em gestão de projetos. Foi utilizado o método stepwise para obter um modelo com o menor número possível variáveis independentes.

Os resultados mostram que com três dimensões da maturidade - Maturidade da Gestão de Custos, Maturidade da Gestão da Integração e do Escopo e Maturidade da Gestão Geral de Projetos - o modelo explica 31,2\% do comportamento da Eficiência do Projeto (Tabela 10).

Tabela 10 - Regressão lears entre Eficiência do Projeto e as dimensões da maturidade

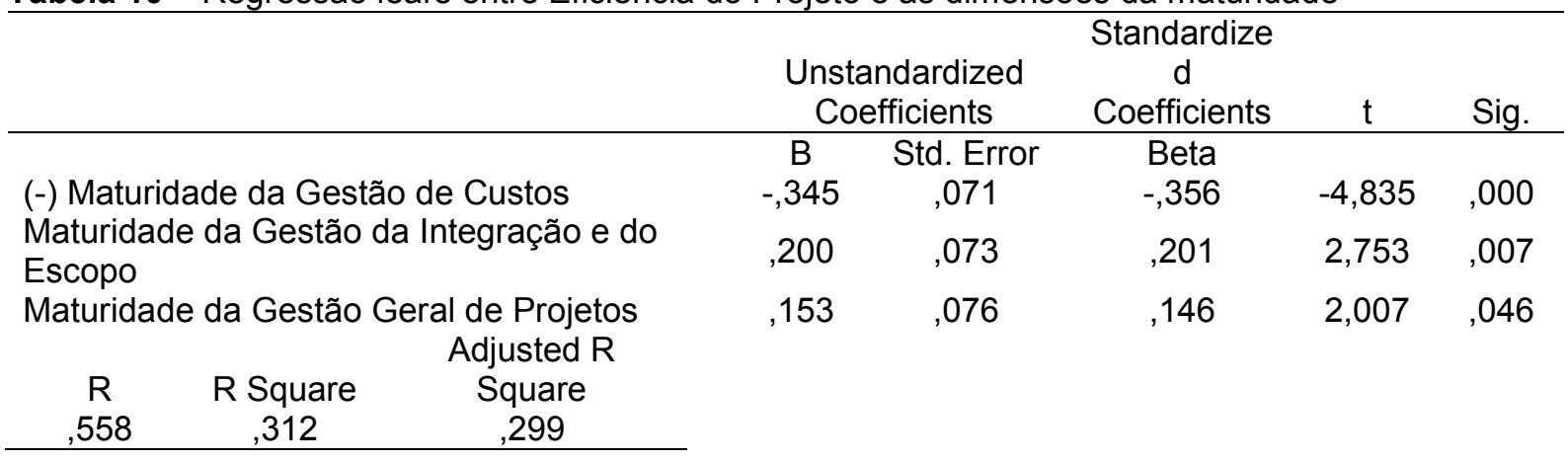

O mesmo procedimento foi realizado para o Impacto no Usuário. Os resultados estão abaixo.

Os resultados (Tabela 1) mostram que com apenas duas dimensões da maturidade - Maturidade da Gestão de Riscos e Maturidade da Gestão de Custos o modelo explica apenas $14,6 \%$ do comportamento do Impacto no Usuário que, em termos práticos, é um valor baixo.

Tabela 11 - Regressão lears entre Eficiência do Projeto e as dimensões da maturidade

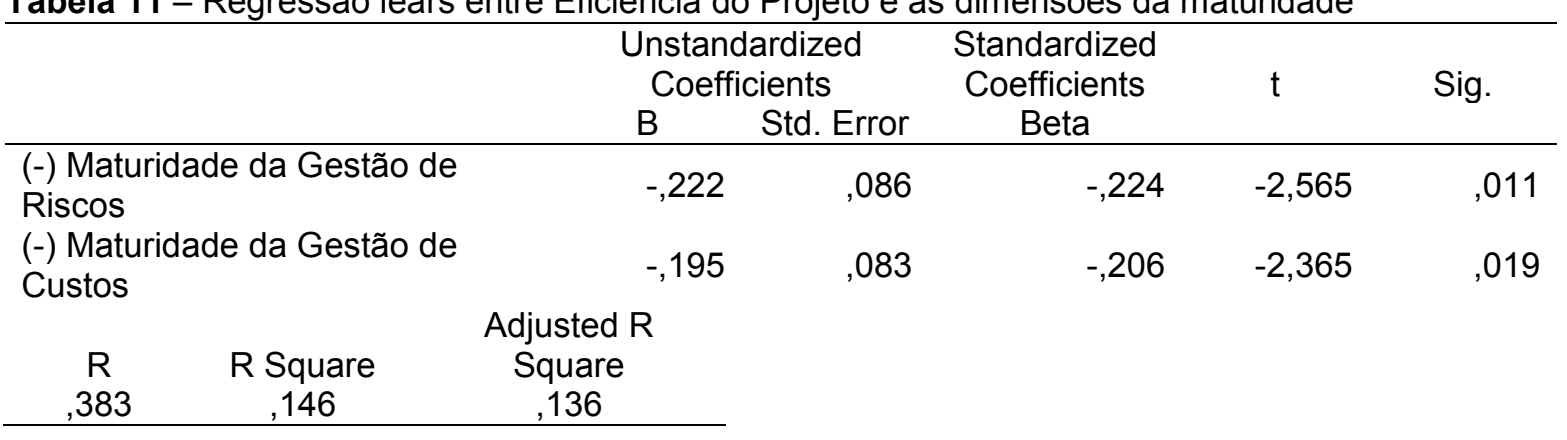

O pequeno número de variáveis independentes (3 e 2) nas regressões diante das seis variáveis candidatas - dimensões da maturidade - deve-se à correlações existentes entre as dimensões da maturidade. 


\section{ANÁLISE DOS DADOS}

$\mathrm{Na}$ amostra foi encontrada correlação estatisticamente significante entre maturidade e desempenho em todas as dimensões destes dois conceitos. Contudo, apesar de estatisticamente significante, as correlações observadas não eram altas. O maior valor encontrado do coeficiente de Pearson foi de 0,522 (entre eficiência do projeto - dimensão do desempenho - e a maturidade interna, que exclui os processos de gestão da aquisição), o que significa que apenas $27 \%$ de comportamento compartilhado entre os dois construtos. Isto sugere que existem outros fatores além da maturidade, não abordados no estudo, que também influenciam o desempenho dos projetos. Mas é importante destacar que, mesmo que baixa, a correlação entre maturidade e desempenho foi observada na amostra. A regressão linear mostrou um resultado semelhante. As dimensões da maturidade explicam 31,2\% com comportamento da Eficiência do Projeto e apenas 14,6\% do comportamento do Impacto no Usuário.

Ao analisar os grupos de grupos de maturidade em gestão de projetos, foi encontrada diferença estatisticamente significante de desempenho. No grupo de maturidade inferior o desempenho dos projetos, tanto em termos de eficiência como em termos de impacto no cliente, foi inferior aos dos outros 2 grupos (maturidade intermediária e superior). Isto sugere que a contribuição da maturidade para o desempenho dos projetos tem um limite. Para organizações mais imaturas um aumento da maturidade em gestão de projetos contribui mais significativamente para a melhoria do desempenho dos projetos. Em organizações não imaturas esta relação não foi observada já que nos grupos de maturidade intermediária e superior foi observado o mesmo nível de desempenho dos projetos.

Assim, parece que organizações diferentes devam buscar níveis de maturidade adequados a sua situação, pelo menos do ponto de vista do nível de desempenho dos projetos. Um grau de maturidade superior ao adequado não iria se traduzir numa maior taxa de sucessos dos projetos. A identificação deste nível adequado de maturidade não foi objeto desta pesquisa e os autores desconhecem na literatura trabalhos desta natureza. Resta aos profissionais de TI o uso da experiência e do conhecimento da organização em que atuam para identificar o investimento adequado na melhoria da maturidade em gestão de projetos. 


\section{CONSIDERAÇÕES FINAIS}

Este artigo visou encontrar evidência empírica da relação maturidade $\mathrm{x}$ desempenho de projetos; Para tanto, apresentou um estudo sobre as relações entre maturidade em gestão de projetos e desempenho de projetos de TI. O levantamento com 185 profissionais da área revelou que existem relações estatisticamente significativas entre as dimensões do desempenho com as diferentes dimensões da maturidade em gestão de projetos identificadas nas análises dos dados. Apesar da evidência estatística, a relevância prática pode ser considerada baixa, conforme revelou a análise dos dados (correlação bivariada e regressão linear múltipla). Isto sugere fortemente que outros elementos condicionantes do desempenho não foram tratados. De fato, a literatura sobre gestão de projetos é vasta em relação aos elementos condicionantes de desempenho dos projetos. Como estes elementos não foram abordados neste estudo, poder-se-ia inferir que a não consideração destes elementos (condicionantes de desempenho encontrados na literatura) seja a causa do baixo poder de determinação encontrado nas análises dos dados.

Pode-se observar também que existe uma diferença (para menor) do desempenho dos projetos nas organizações de maturidade inferior em relação aos outros grupos (de maturidade intermediária e maturidade superior). Desta forma, como uma consequência deste trabalho para os profissionais da área, é que as organizações com um baixo nível de formalização de seus processos de gestão de projetos são as que mais têm a ganhar com os esforços de aumento da maturidade em gestão projetos.

Como limitações deste trabalho, pode-se citar a simplificação que foi feita acerca da forma como foi avaliado o conceito de maturidade, fundamentalmente se baseando na formalização de processos e o fato da amostra não ser de fato realmente aleatória. Do ponto de vista estatístico, dados extraídos de amostras não aleatórias não permitem inferências sobre a população. Contudo, esta simplificação tornou o estudo viável.

Como desdobramento futuro deste estudo, pretende-se avaliar como a maturidade afeta a relação (importância relativa) dos elementos condicionantes de desempenho com o desempenho dos projetos de TI. Um estudo desta natureza ajudaria a compreender melhor os (eventuais, outros benefícios da maturidade em gestão de projetos. Além disto, outros estudos que operacionalizassem os conceitos 
de maturidade e desempenho de forma mais ampla poderiam contribuir para um melhor entendimento destes dois conceitos.

\section{REFERÊNCIAS}

ANTONIONI, L. e ROSA, N. B. Qualidade em software: manual de aplicação da ISO 9000. São Paulo: Makron Books, 1995.

BACCARINI, David The Logical Framework Method for Defining Project Success In: International Journal of Project Management v. 30, n. 4, p. 25-32, 1999.

BALOCCO, R.; MAINETTI, S.; RANGONE, A. Innovare e competere con le ICT. II Sole 24 Ore, Milano, 2006, 177p.

BAKER, Bruce N., MURPHY, David C. e FISHER, Dalmar "Factors affecting project success" In: CLELAND, D. I. \& KING, W. R. Project management handbook. New York: John Wiley, 1983.

BENKO, C.; MCFARLAN, F.W. Connecting the dots. Harvard Business School Press. Boston, 246 p., 2003.

BROWN, J. R.; GOOLSBEE, A. Does the internet make markets more competitive? evidence from the life insurance industry. Journal of Political Economy, v.110, n.3, p. 481-507, 2002.

COOKE-DAVIES, T. The real success factors on projects. International Journal of Project Management, v. 20, p. 185-190, 2000.

FARRELL, D. The real new economy . Harvard Business Review, 9 p., October 2003.

FINCHER, A. and LEVIN, G., Project management maturity model: project Management Institute 28th Annual Seminar/Symposium, Chicago, III., 1997, pp. 4855.

GEREFFI,G. Beyond the producer-driven/buyer-driven dichotomy: the evolution of global value chains in the internet era. IDS Bulletin, v.32, n. 3, p.30-40, July 2001.

GOLDSMITH, L. Approaches towards effective project management, Project Management Maturity Model. Project Management Institute 28th Annual Seminar/Symposium, Chicago, III., 1997. p. 49-54.

HAIR JR, J. F. Análise multivariada de dados. Porto Alegre: Bookman, 2009

HARTMAN, F. T. \& SKULMOSKI, G. Project management maturity. Project Management Journal, 1998. p. 74-78 
HAYES, R.; UPTON, D.; PISANO, G.; WHEELWRIGHT, S. Produção, estratégia e tecnologia: em busca da vantagem competitive. Bookman, Porto Alegre. 384 p., 2008.

IBBS, W. e KWAK, Y.H. The benefits of project management: financial and organizational rewards to corporations. Project Management Institute. Sylvia, N.C. , 1997.

IBBS, W. e KWAK, Y.H. Assessing Project Management Maturity. Project Management Journal, v. 31, n. 1, p. 32-43, March 2000.

KALANTJAKOSN. J. Assessing Organizational Project Management Maturity. Proceedings of the Project Management Institute Annual Seminars \& Symposium, USA: Nashville, Tennessee, Nov. 2001.

LIM, C. S. e MOHAMED, M. Z. Criteria of project success: an exploratory reexamination. In International Journal of Project Management, v. 17, n. 4, p. 243248, 1999.

LOCKE, D. Project Management. New York: St Martins Press, 1984.

MAXIMIANO, A. C. A. E RABECHINI Jr, R. Maturidade em gestão de projetos análise de um caso proposição de um modelo. SIMPÓSIO D GESTÃO DA INOVAÇÃO TECNOLÓGICA. 23., 2002. Anais... Salvador, 2002.

McGRATH, Michael E. Revving up product development. Electronic Business Magazine, p. 36, january 1998.

MUNNS, A. K. \& BJEIRMI, B. F. The role of project management in achieving project success. In: International Journal of Project Management, v.14, n. 2, p. 81-87, 1997.

MORAES, R. O.; KRUGLIANSKAS, I. Projetos de TI: maturidade $x$ desempenho. Revista de Administração e Inovação, v.7, n. 2, 2010.

MORAES, R. O. Condicionantes de desempenho dos projetos de software e a influência da maturidade em gestão de projetos. 2004. Tese (Doutorado em Administração de Empresas). Programa de Pós-Graduação em Administração de Empresas. Faculdade de Economia, Administração e Contabilidade da Universidade de São Paulo, São Paulo - SP.

MORAES, R. O.; LAURINDO, F. J. B. Multidimensionalidade da maturidade em gestão de projetos de sistemas de informação. Produto \& Produção, v.9, p.1 - 18, 2008.

MORAES, R. O. ; LAURINDO, F. J. B. Avaliação de resultados de projetos de TI. In. ENCONTRO NACIONAL DE ENGENHARIA DE PRODUÇÃO, 30,. 2010. São Carlos. Anais... São Carlos: ABEPRO, 2010.

PAULK, Marc C. et all The capability maturity model: guidelines for improving the software process Addison-Wesley, 1994. 
PINTO, J. K. \& SLEVIN, D. P. Project Success : Definitions and measurement techniques. International Journal of Project Management, 1988.

PINTO, J. K. \& SLEVIN, D. P. Critical success factors across the project life cycle. International Journal of Project Management , 1986.

PMBOK A guide to the project management body of knowledge. PMI - Project Management Institute , 2008.

PORTER, M.E. Strategy and the internet. Harvard Business Review, p.63-78, March, 2001.

PRAHALAD, C.K. \& KRISHNAN, M.S. A Nova Era da Inovação. Rio de Janeiro: Elsevier, 2008.

REMY, R. Adding focus to improvement efforts with PM3.PM Network, July, 1997.

SHENHAR, A. et al. Project success: a multidimensional strategic concept. Long Range Planning, n. 34, p. 699-725, 2001.

SCHLICHTER, J. PMI's Organizational project management maturity model: Emerging Standards. PROJECT MANAGEMENT INSTITUTE ANNUAL SEMINARS \& SYMPOSIUM. Proceedings... USA: Nashville, Tennessee, Nov. 2001.

TAPSCOTT, D. Rethinking strategy in a networked world. Strategy + Business, issue 24, 8p., 2001.

VENKATRAMAN, N.;HENDERSON, J. C, Real strategies for virtual organizing. Sloan management Review,v. 40, n.1, p. 33-48, Fall 1998.

YASSINE, A.; KIM, K.C.; ROEMER, T. \& HOLWEG, M. Investigating the role of IT in customized product design. Production Planning \& Control, v. 15, n. 4, p. 422-434, June 2004.

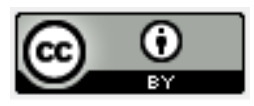

